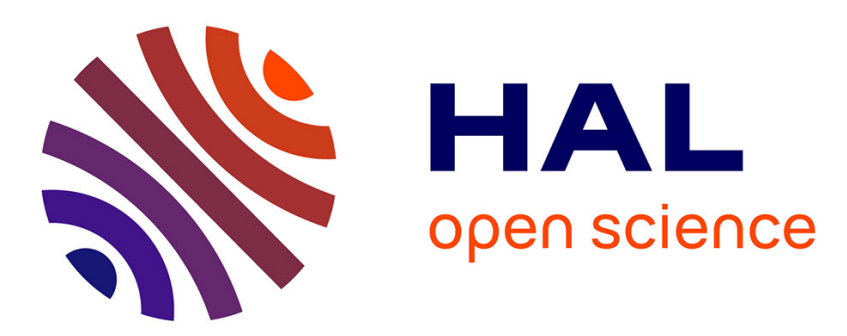

\title{
Instantaneous optimal control of friction dominated flow in a gas-network
}

Günter Leugering, Gisèle Mophou

\section{To cite this version:}

Günter Leugering, Gisèle Mophou. Instantaneous optimal control of friction dominated flow in a gasnetwork. DFG-AIMS Workshop on Shape Optimization, Homogenization and Control., Mar 2017, Mbour, Senegal. hal-02548134

\section{HAL Id: hal-02548134 \\ https://hal.science/hal-02548134}

Submitted on 20 Apr 2020

HAL is a multi-disciplinary open access archive for the deposit and dissemination of scientific research documents, whether they are published or not. The documents may come from teaching and research institutions in France or abroad, or from public or private research centers.
L'archive ouverte pluridisciplinaire HAL, est destinée au dépôt et à la diffusion de documents scientifiques de niveau recherche, publiés ou non, émanant des établissements d'enseignement et de recherche français ou étrangers, des laboratoires publics ou privés. 


\title{
Instantaneous optimal control of friction dom- inated flow in a gas-network
}

\author{
Günter Leugering and Gisèle Mophou
}

\begin{abstract}
We consider optimal control problems for the flow of gas in a pipe network. The equations of motions are taken to be represented by a nonlinear model derived from asemi-linear approximation of the fully nonlinear isothermal Euler gas equations. We formulate an optimal control problem on a given network and introduce a time discretization thereof. We then study the well-posedness of the corresponding time-discrete optimal control problem. In order to further reduce the complexity, we consider an instantaneous control strategy. This involves a p-Laplace-type problem on the graph with $p=\frac{3}{2}$. We prove well-posedness, existence of optimal controls and derive a first order optimality condition.
\end{abstract}

Mathematics Subject Classification (2010). 35J70,49J20,49J45,93C73.

Keywords. Optimal control, gas networks, p-Laplace problem on a graph, optimality system.

\section{Introduction}

\subsection{Modeling of gas flow in a single pipe}

The Euler equations are given by a system of nonlinear hyperbolic partial differential equations (PDEs) which represent the motion of a compressible non-viscous fluid or a gas. They consist of the continuity equation, the balance of moments and the energy equation. The full set of equations is given by (siehe $[4,9,10,12])$ Let $\rho$ denote the density, $v$ the velocity of the

The first author was supported by the DFG-TRR 154 "Modellierung Simulation und Optimierung am Beispiel von Gasnetzwerken" (TPA05), the second author was supported by the Alexander von Humboldt foundation, under the programme financed by the BMBF entitled "German research Chairs" . 
gas and $p$ the pressure. We further denote $g$ the gravitational constant, $\lambda$ the friction coefficient of the pipe, $D$ the diameter, $a$ the area of the cross section. The state variables of the system are $\rho$, the flux $q=\rho v$. We also denote $c$ the the speed of sound, i.e. $c^{2}=\frac{\partial p}{\partial \rho}$ (for constant entropy). For natural gas we have $340 \frac{\mathrm{m}}{\mathrm{s}}$. In particular, in the subsonic case $(|v|<c)$, the one which we consider in the sequel, two boundary conditions have to be imposed on the left end and one at the right end of the pipe. We consider here the isothermal case only. Thus, for horizontal pipes

$$
\begin{aligned}
\frac{\partial \rho}{\partial t}+\frac{\partial}{\partial x}(\rho v) & =0 \\
\frac{\partial}{\partial t}(\rho v)+\frac{\partial}{\partial x}\left(p+\rho v^{2}\right) & =-\frac{\lambda}{2 D} \rho v|v| .
\end{aligned}
$$

In the particular case, where the we have a constant speed of sound $c=\sqrt{\frac{p}{\rho}}$, for small velocities $|v| \ll c$, we arrive at the semi-linear model

$$
\begin{aligned}
& \frac{\partial \rho}{\partial t}+\frac{\partial}{\partial x}(\rho v)=0 \\
& \frac{\partial}{\partial t}(\rho v)+\frac{\partial p}{\partial x}=-\frac{\lambda}{2 D} \rho v|v| .
\end{aligned}
$$

If we further neglect the inertia with respect to the flux and introduce $q=\rho v a$, we arrive at

$$
\begin{aligned}
\frac{\partial p}{\partial t}+\frac{c^{2}}{a} \frac{\partial}{\partial x} q & =0 \\
\frac{\partial p^{2}}{\partial x} & =-\frac{\lambda c^{2}}{D a^{2}} q|q|=:-\gamma^{2} q|q| .
\end{aligned}
$$

We now set $y:=p^{2}$ and obtain from the second equation in (1.3)

$$
q=-\frac{1}{\gamma} \frac{\frac{\partial y}{\partial x}}{\sqrt{\left|\frac{\partial y}{\partial x}\right|}} .
$$

With $\alpha:=\frac{\gamma a}{c}$ we obtain

$$
\alpha \frac{\partial}{\partial t} \frac{y}{\sqrt{|y|}}-\frac{\partial}{\partial x} \frac{\frac{\partial y}{\partial x}}{\sqrt{\left|\frac{\partial y}{\partial x}\right|}}=0 .
$$

We introduce the monotone function $\beta(s):=\frac{s}{\sqrt{|s|}}$. With this (1.4) reads as

$$
\alpha \frac{\partial}{\partial t} \beta(y)-\frac{\partial}{\partial x} \beta\left(\frac{\partial y}{\partial x}\right)=0 .
$$


It is also possible to write this down in the p-Laplace format: (1.4) reads as

$$
\alpha \frac{\partial}{\partial t}\left(|y|^{p-2} y\right)-\frac{\partial}{\partial x}\left(\left|\frac{\partial y}{\partial x}\right|^{p-2} \frac{\partial y}{\partial x}\right)=0
$$

where $p=\frac{3}{2}$. Equation (1.6) has come to be known as doubly nonlinear parabolic equation of p-Laplace type. See [11]. Notice that $p<2$ and that the system is, therefore, singular for $\frac{\partial}{\partial x} y(x)=0$. For $p>2$ such equations exhibit instead degeneration. Equations similar to (1.5) have been considered in the literature, see e.g. $[2,3]$. In this contribution, we aim at a discussion of such equations together with optimal control problems on networks. A more recent study of doubly nonlinear parabolic equations in the context of friction dominated flow has been provided in [1]. Equations of the type (1.5) are known to exhibit positive solutions, finite speed of propagation and satisfy a maximum principle. As a matter of fact, to the best knowledge of the authors, there are no studies on optimal control of such systems on general graphs available from the literature. Therefore, these notes can be seen as the first attempt in that direction. We refer to a forthcoming publication, where the additional properties mentioend, full proofs and numerical analysis are provided [8].

\subsection{Network modeling}

Let $G=(V, E)$ denote the graph of the gas network with vertices (nodes) $V=\left\{n_{1}, n_{2}, \ldots, n_{|V|}\right\}=\left\{n_{j} \mid j \in \mathcal{J}\right.$ an edges $E=\left\{e_{1}, e_{2}, \ldots, e_{|E|}\right\}=$ $\left\{e_{i} \mid i \in \mathcal{I}\right\}$. For the sake of uniqueness, we associate to each edge a direction.

$$
d_{i j}=\left\{\begin{array}{cc}
-1, & \text { if node } n_{j} \text { if the the edge } e_{i} \text { starts at node } n_{j} e_{i}, \\
+1, & \text { if node } n_{j} \text { if the edges } e_{i} \text { end at node } n_{j} e_{i}, \\
0, & \text { else. }
\end{array}\right.
$$

The pressure variables $y_{i}\left(n_{j}\right)$ coincide for all $i \in \mathcal{I}_{j}:=\left\{i \in 1, \ldots E \mid d_{i j} \neq 0\right\}$. We express the transmission conditions at the nodes in the following way. We introduce the edge degree $d_{j}:=\left|\mathcal{I}_{j}\right|$. Then the continuity conditions read as follows

$$
y_{i}\left(n_{j}, t\right)=y_{k}\left(n_{j}, t\right), \forall i, k \in \mathcal{I}_{j}, d_{j}>1 .
$$

The nodal balance equation for the fluxes can be written as the classical Kirchhoff-type condition

$$
\sum_{i \in \mathcal{I}_{j}} d_{i j} \beta\left(\partial_{x} y_{i}\left(n_{j}, t\right)\right)=0, d_{j}>1
$$

We use the $d_{j}$ in order to decompose the index set for nodes $\mathcal{J}$ into $\mathcal{J}=$ $\mathcal{J}^{M} \cup \mathcal{J}^{S}$, where $\mathcal{J}^{M}=\left\{j \in \mathcal{J} \mid d_{j}>1\right\}$ represents the multiple nodes and $\mathcal{J}^{S}=\left\{j \in \mathcal{J} \mid d_{j}=1\right\}$ the simple nodes. According to Dirichlet or 
Neumann boundary conditions a the simple nodes, we further decompose $\left.\mathcal{J}^{S}=\mathcal{J}_{D}^{S} \cup \mathcal{J}_{N}^{S}\right\}$. We summarize the equations as follows:

$$
\begin{gathered}
\alpha_{i} \partial_{t} \beta\left(y_{i}(x, t)\right)+\partial_{x}\left(\beta\left(\partial_{x} y_{i}(x, t)\right)\right)=0, i \in \mathcal{I}, x \in\left(0, \ell_{i}\right), t \in(0, T) \\
y_{i}\left(n_{j}, t\right)=y_{k}\left(n_{j}, t\right), \forall i, k \in \mathcal{I}_{j}, j \in \mathcal{J}^{M}, t \in(0, T) \\
\sum_{i \in \mathcal{I}_{j}} d_{i j} \beta\left(\partial_{x} y_{i}\left(n_{j}, t\right)\right)=0, j \in \mathcal{J}^{M}, t \in(0, T) \\
y_{i}\left(n_{j}, t\right)=0, i \in \mathcal{I}_{j}, j \in \mathcal{J}_{D}^{S}, t \in(0, T) \\
d_{i j} \beta\left(\partial_{x} y_{i}\left(n_{j}, t\right)=u_{j}(t), i \in \mathcal{I}_{j}, j \in \mathcal{J}_{N}^{S}, t \in(0, T)\right. \\
p_{i}(x, 0)=p_{i, 0}(x), q_{i}(x, 0)=q_{i 0}(x), x \in\left(0, \ell_{i}\right)
\end{gathered}
$$

To the best knowledge of the authors, problem (1.9), no published result seems to be available.

Optimal control problems and outline. We are now in the position to formulate optimal control problems on the level of the gas networks. We first describe the general format for an optimal control problem associated with the semi-linear model equations. This involves a cost function that assigns to admissible each pair $(y, u)$ a 'cost' $I(y, u)$, which is represented on each individual edge by a contribution on the state $I_{i}(y)$ and the controls acting at simple nodes. The typical example, the one that we will use in the sequel is given by

$$
\begin{gathered}
I_{i}\left(y_{i}\right)(x):=\frac{1}{p}\left|y_{i}(x)-y_{i}^{d}(x)\right|^{p}, x \in\left(0, \ell_{i}\right), p \in\left\{\frac{3}{2}, 2\right\} . \\
\min _{(y, u) \in \Xi} I(y, u):=\sum_{i \in \mathcal{I}} \int_{0}^{T} \int_{0}^{\ell_{i}} I_{i}\left(y_{i}\right) d x d t+\frac{\nu}{2} \sum_{j \in \mathcal{J}_{N}^{S}} \int_{0}^{T}\left|u_{j}(t)\right|^{2} d t \\
\quad \text { s.t. } \\
\quad(y, u) \text { satisfies }(1.9), \\
\Xi:=\left\{(y, u): \underline{y} i \leq y_{i} \leq \bar{y}_{i}, i \in \mathcal{I}, \underline{u_{j}} \leq u_{j} \leq \overline{u_{j}}, j \in \mathcal{J}_{N}^{S}\right\} .
\end{gathered}
$$

In (1.11), the quantities $\underline{y}_{i}, \bar{y}_{i}$ are given constants that determine the feasible pressures and flows in the pipe $i$, while $\underline{u}_{i}, \bar{u}_{i}$ describe control constraints. In the continuous-time case the inequalities are considered as being satisfied for all times and everywhere along the pipes. Due to limitations in space, we will not consider state- and control constraints here and refer instead to a forthcoming article [8]. , Instead, we just penalize the control costs using $\nu>0$. Moreover, we will restrict ourselves to time discretizations of (1.10) and, in fact, to the instantaneous control regime that has come to be known also as rolling horizon problem. 


\subsection{Time discretization}

We, therefore, consider the time discretization (1.9) such that $[0, T]$ is decomposed into break points $t_{0}=0<t_{1}<\cdots<t_{N}=T$ with widths ${ }^{\Delta} t_{n}:=t_{n+1}-t_{n}, n=0, \ldots, N-1$ (we use $N+1$ as the number of break points which is not related to $N$ as indicating Neumann conditions). Accordingly, we denote $p_{i}\left(x, t_{n}\right):=p_{i, n}(x), q_{i}\left(x, t_{n}\right):=q_{i, n}(x), n=0, \ldots, N-1$. We consider a semi-implicit Euler scheme which takes $p_{i}$ in the friction term in an explicit manner.

$$
\begin{aligned}
\frac{1}{\Delta t} \beta\left(y_{i, n+1}\right)(x)-\partial_{x}\left(\beta\left(\partial_{x} y_{i, n+1}(x)\right)\right) & =\frac{1}{\Delta t} \beta\left(y_{i, n}\right)(x), i \in \mathcal{I}, x \in\left(0, \ell_{i}\right) \\
y_{i, n+1}\left(n_{j}\right) & =y_{k, n+1}\left(n_{j}\right), \forall i, k \in \mathcal{I}_{j}, j \in \mathcal{J}^{M} \\
\sum_{i \in \mathcal{I}_{j}} d_{i j} \beta\left(\partial_{x} y_{i, n+1}\right)\left(n_{j}\right) & =0, j \in \mathcal{J}^{M} \\
\beta\left(\partial_{x} y_{i, n+1}\right)\left(n_{j}\right) & =u_{j, n+1}, d_{j}=1, i \in \mathcal{I}_{j}, j \in \mathcal{J}_{N}^{S} \\
y_{i, n+1}\left(n_{j}\right)=0, i & \in \mathcal{I}_{j}, j \in \mathcal{J}_{D}^{S} \\
y_{i, 0}(x)=y_{i, 0}(x), i & \in \mathcal{I}, x \in\left(0, \ell_{i}\right), n=1, \ldots, N-1 .
\end{aligned}
$$

We then obtain the optimal control problem on the time-discrete level:

$$
\begin{aligned}
\min _{(y, u)} I(y, u):= & \sum_{i \in \mathcal{I}} \sum_{n=1}^{N} \int_{0}^{\ell_{i}} I_{i}\left(y_{i, n}\right) d x+\frac{\nu}{2} \sum_{j \in \mathcal{J}_{N}^{S}} \sum_{n=1}^{N}\left|u_{j}(n)\right|^{2} \\
& \text { s.t. } \\
& (y, u) \text { satisfies (1.12) }
\end{aligned}
$$

It is clear that (1.13) involves all time steps in the cost functional. We would like to reduce the complexity of the problem even further. To this aim we consider the instantaneous control regime. This amount to reducing the sums in the cost function of (1.13) to the time-level $t_{n+1}$. This strategy has also come to be known as rolling horizon approach, the simplest case of the moving horizon paradigm. Thus, for each $n=1, \ldots, N-1$ and given $y_{i, n}$, we consider the problems

$$
\begin{aligned}
& \min _{(y, u)} I(y, u):=\sum_{i \in \mathcal{I}} \int_{0}^{\ell_{i}} I_{i}\left(y_{i}\right) d x+\frac{\nu}{2} \sum_{j \in \mathcal{J}_{N}^{S}}\left|u_{j}\right|^{2} \\
& \text { s.t. } \\
& (y, u) \text { satisfies }(1.12) \text { at time level } n+1 .
\end{aligned}
$$



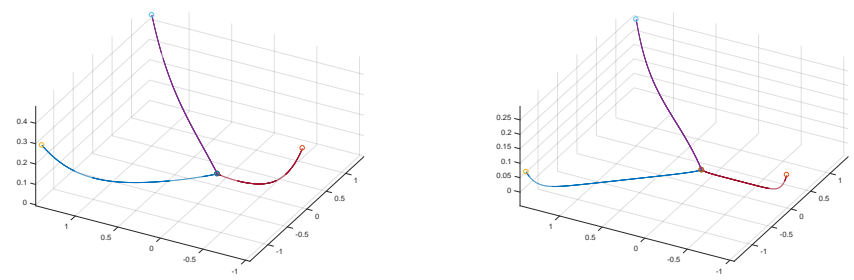

FIGURE 1. left: $\alpha=10, \gamma=1, f_{i}=10$; right: $\alpha=10$, $\gamma=100, f_{i}=10$

It is now convenient to discard the actual time level $n+1$ and redefine the states at the former time as input data. To this end, we replace $\alpha_{i}:=\frac{1}{\Delta t}$, $f_{i}^{1}:=\alpha_{i} \beta\left(y_{i, n}\right)$, rewrite $(1.12)$ as

$$
\begin{aligned}
& \alpha_{i} \beta\left(y_{i}\right)(x)-\partial_{x}\left(\beta\left(\partial_{x} y_{i}(x)\right)\right)=f_{i}^{1}(x), i \in \mathcal{I}, x \in\left(0, \ell_{i}\right) \\
& y_{i}\left(n_{j}\right)=y_{k}\left(n_{j}\right), \forall i, k \in \mathcal{I}_{j}, j \in \mathcal{J}^{M} \\
& \sum_{i \in \mathcal{I}_{j}} d_{i j} \beta\left(\partial_{x} y_{i}\right)\left(n_{j}\right)=0, j \in \mathcal{J}^{M} \\
& \beta\left(\partial_{x} y_{i}\right)\left(n_{j}\right)=u_{j}, d_{j}=1, i \in \mathcal{I}_{j}, j \in \mathcal{J}_{N}^{S} \\
& y_{i}\left(n_{j}\right)=0, i \in \mathcal{I}_{j}, j \in \mathcal{J}_{D}^{S}
\end{aligned}
$$

Example. We consider a star-graph with three edges (tripod). We take four numerical examples in order to illustrate the behavior of the system with respect to parameter changes. In Fig. 1.1, we take the parameters $\alpha_{i}=10$, $\gamma_{i}=1, f_{i}=10$; right: $\alpha_{i}=10, \gamma_{i}=100, f_{i}=10$, where $\gamma$ puts a weight on the nonlinearity. In the second Fig. 1.2, we take all parameters equal and set $\alpha=100, \gamma=1, f_{i}=10$; right: $\alpha=1, \gamma=0.1, f_{i}=10$. The calculations are done with the routine bvp 4 c.m from MATLAB (R2017a). 

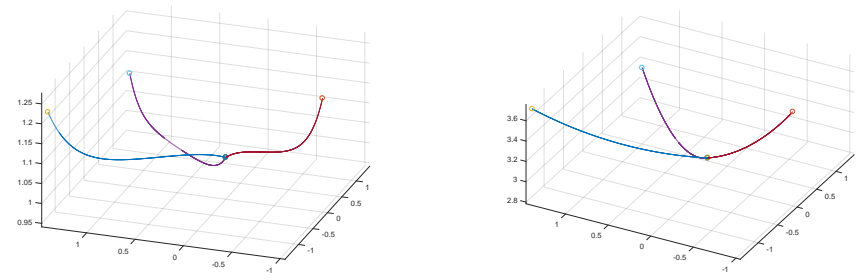

FiguRE 2. left: $\alpha=100, \gamma=1, f_{i}=10$; right: $\alpha=1$, $\gamma=0.1, f_{i}=10$

We now consider in the rest of the paper the following optimal control problem:

$$
\begin{aligned}
\min _{(y, u)} I(y, u):= & \sum_{i \in \mathcal{I}} \int_{0}^{\ell_{i}} I_{i}\left(y_{i}\right) d x+\frac{\nu}{2} \sum_{j \in \mathcal{J}_{N}^{S}}\left|u_{j}\right|^{2} \\
\text { s.t. } &
\end{aligned}
$$

$(y, u)$ satisfies $(1.15)$. 


\section{Well-posedness}

We begin our analysis of (1.15) with a Lagrange identity. To this end, we multiply the equation by a test function $\phi$ and integrate by parts.

$$
\begin{aligned}
0= & \sum_{i \in \mathcal{I}} \int_{0}^{\ell_{i}}\left(\alpha_{i} \beta\left(y_{i}\right)-\partial_{x}\left(\beta\left(\partial_{x} y_{i}\right)\right)-f_{i}\right) \phi_{i} d x \\
= & \sum_{i \in \mathcal{I}} \int_{0}^{\ell_{i}}\left(\alpha_{i} \beta\left(y_{i}\right) \phi_{i}+\left(\beta\left(\partial_{x} y_{i}\right)\right) \partial_{x} \phi_{i}-f_{i} \phi_{i}\right) \phi_{i} d x \\
& -\sum_{j \in \mathcal{J}^{M}} \sum_{i \in \mathcal{I}_{j}} d_{i j} \beta\left(\partial_{x} y_{i}\left(n_{j}\right)\right) \phi_{i}\left(n_{j}\right)-\sum_{j \in \mathcal{J}_{N}^{S}} \sum_{i \in \mathcal{I}_{j}} u_{j} \phi_{i}\left(n_{j}\right)
\end{aligned}
$$

If the test function satisfies the nodal conditions of order zero, we arrive at the variational equation which serves for the proper definition of weak solutions

$$
\sum_{i \in \mathcal{I}} \int_{0}^{\ell_{i}}\left(\alpha_{i} \beta\left(y_{i}\right) \phi_{i}+\left(\beta\left(\partial_{x} y_{i}\right)\right) \partial_{x} \phi_{i}\right) \phi_{i} d x=\sum_{j \in \mathcal{J}_{N}^{S}} \sum_{i \in \mathcal{I}_{j}} u_{j} \phi_{i}\left(n_{j}\right)+\sum_{i \in \mathcal{I}} \int_{0}^{\ell_{i}} f_{i} \phi_{i} .
$$

For the sake of simpler notation, we switch freely between the formulation involving $\beta(\cdot)$ and the explicit definition of $\beta(s)=\frac{s}{\sqrt{|s|}}$. Therefore, in our analysis $p=\frac{3}{2}$, even though, in many ways the results produced here are valid for general $p \leq 2$. For the general case, we refer to [8]. It is clear then that the dual space of $L^{p}\left(0, \ell_{i}\right)$ is $L^{q}\left(0, \ell_{i}\right)=L^{3}\left(0, \ell_{i}\right)$. The mapping $\beta(\cdot)$ satisfies

$$
\beta: L^{p}\left(0, \ell_{i}\right) \rightarrow L^{q}\left(0, \ell_{i}\right), \text { is bounded }
$$

Indeed, $\|\beta(\phi)\|_{L^{q}\left(0, \ell_{i}\right)} \leq\|\phi\|_{L^{p}\left(0, \ell_{i}\right)}^{\frac{1}{2}}$. This applies of course also to $\beta\left(\partial_{x} \phi\right)$. We now define the strong version of the guiding operator $\mathcal{A}$ :

$$
\begin{aligned}
(\mathcal{A} y)_{i \in \mathcal{I}}:= & \left(\alpha_{i} \beta\left(y_{i}\right)-\partial_{x}\left(\beta\left(\partial_{x} y_{i}\right)\right)\right)_{i \in \mathcal{I}} \\
D(\mathcal{A}):=\{ & \left(y_{i}\right)_{i \in \mathcal{I}} \in \Pi_{i \in \mathcal{I}} W^{1, p}\left(0, \ell_{i}\right) \mid \partial_{x} \beta\left(\partial_{x} y_{i}\right) \in L^{q}\left(0, \ell_{i}\right), \\
& \sum_{i \in \mathcal{I}_{j}} d_{i j} \beta\left(\partial_{x} y_{i}\left(n_{j}\right)\right) \phi_{i}\left(n_{j}\right)=0, \\
& y_{i}\left(n_{j}\right)=y_{k}\left(n_{j}\right), i, k \in \mathcal{I}_{j}, j \in \mathcal{J}^{M}, \\
& \left.y_{i}\left(n_{j}\right)=0, i \in \mathcal{I}_{j}, j \in \mathcal{J}_{D}^{S}, \beta\left(\partial_{x} y_{i}\left(n_{j}\right)\right)=0, i \in \mathcal{I}_{j}, j \in \mathcal{J}_{N}^{S}\right\}
\end{aligned}
$$


As for the weak formulation, we define the energy space

$$
\begin{aligned}
V:=\left\{\left(y_{i}\right)_{i \in \mathcal{I}} \in \Pi_{i \in \mathcal{I}} W^{1, p}\left(0, \ell_{i}\right) \mid y_{i}\left(n_{j}\right)=y_{k}\left(n_{j}\right), i, k \in \mathcal{I}_{j}, j \in \mathcal{J}^{M},\right. \\
\left.y_{i}\left(n_{j}\right)=0, i \in \mathcal{I}_{j}, j \in \mathcal{J}_{D}^{S}\right\},
\end{aligned}
$$

where $\|\phi\|_{V}:=\sum_{i \in \mathcal{I}}\left\|\phi_{i}\right\|_{W^{1, p}\left(0, \ell_{i}\right)}$. Thus, for $y \in D(\mathcal{A})$ we obtain

$$
\begin{aligned}
\langle\mathcal{A}(y), \phi\rangle & :=\sum_{i \in \mathcal{I}} \int_{0}^{\ell_{i}}\left(\alpha_{i} \beta\left(y_{i}\right)-\partial_{x}\left(\beta\left(\partial_{x} y_{i}\right)\right) \phi_{i} d x\right. \\
& =\sum_{i \in \mathcal{I}_{j}} \int_{0}^{\ell_{i}}\left(\alpha_{i} \beta\left(y_{i}\right) \phi_{i}+\left(\beta\left(\partial_{x} y_{i}\right)\right) \partial_{x} \phi_{i}\right) \phi_{i} d x \\
& =\langle\mathcal{A}(y), \phi\rangle_{V^{*}, V}, \forall \phi \in V
\end{aligned}
$$

Using the boundedness of $\beta(\cdot)$, we infer that $\mathcal{A}: V \rightarrow V^{*}$ is bounded as a nonlinear operator. Indeed, for an open neighbourhood $U(0)$ of 0 in $V$,

$$
\begin{aligned}
& \langle\mathcal{A}(y), \phi\rangle_{V^{*}, V} \\
& \leq C \sum_{i \in \mathcal{I}}\left\|y_{i}\right\|_{W^{1, p}\left(0, \ell_{i}\right)}\|\phi\|_{W^{1, p\left(0, \ell_{i}\right)}} \leq C\|\phi\|_{V}, \forall y \in U(0) \subset V, \phi \in V .
\end{aligned}
$$

We obtain the energy form

$$
\langle\mathcal{A}(y), y\rangle=\sum_{i \in \mathcal{I}_{j}} \int_{0}^{\ell_{i}} \alpha_{i}\left|y_{i}\right|^{p}+\left|\partial_{x} y_{i}\right|^{p} d x \geq \underline{\alpha}\|y\|_{V}^{p} .
$$

In order to further investigate the properties of $\mathcal{A}$, we recall ( [5], [7])

$$
\left(|a|^{p} a-|b|^{p} b\right)(a-b) \geq(1+|a|+|b|)^{p-2}|a-b|^{2}, 1 \leq p \leq 2 .
$$

From this it follows

$$
\langle\mathcal{A}(y)-\mathcal{A}(z), y-z\rangle_{V^{*}, V} \geq c\|y-z\|_{V}^{2}>0, \forall y, z \in V,
$$

for some $c>0$. The inequality (2.9) implies that $\mathcal{A}$ is strictly monotone. We also easily verify

$$
\frac{\langle\mathcal{A}(y), y\rangle_{V^{*}, V}}{\|y\|_{V}} \rightarrow \infty, \text { as }\|y\|_{V} \rightarrow \infty,
$$

i.e. the coercivity of $\mathcal{A}$. We have

$$
\begin{aligned}
&\left\langle\mathcal{A}\left(y^{k}\right)-\right.\left.\mathcal{A}\left(y^{0}\right), \phi\right\rangle_{V^{*}, V} \\
&=\sum_{i \in \mathcal{I}_{j}} \int_{0}^{\ell_{i}} \alpha_{i}\left(\beta\left(y_{i}^{k}\right)-\beta\left(y_{i}^{0}\right)\right) \phi_{i}+\left(\beta\left(\partial_{x} y_{i}^{k}\right)-\beta\left(\partial_{x} y_{i}^{0}\right)\right) \partial_{x} \phi_{i} d x \rightarrow 0,
\end{aligned}
$$


as $y^{k} \rightarrow y^{0}$ in $V$ for $k \rightarrow \infty$. This shows that $\mathcal{A}$ is demi-continuous. Summarizing, we have shown that $\mathcal{A}$ is demi-continuous, coercive and strictly, monotone. Applying the classical Brezis' result (see e.g. [11]), we infer

$$
\forall f \in V^{*} \exists ! y \in V: \mathcal{A}(y)=f .
$$

The sense of being a solution is weak, as $y \in V$. Clearly, more regular data imply strong solutions. If we consider the boundary controls as

$$
f_{u}(\phi):=\sum_{j \in \mathcal{J}_{N}^{S}} \sum_{i \in \mathcal{I}_{j}} u_{j} \delta_{n_{j}}(\phi),
$$

then $f_{u} \in V^{*}$ and we obtain the following

Theorem 2.1. For $f \in \Pi_{i \in \mathcal{I}} L^{3}\left(0, \ell_{i}\right), u \in \mathbb{R}^{\left|\mathcal{J}_{N}^{S}\right|}$, problem (1.15) admits a unique weak solution $y \in V$.

Even though, the mapping $\beta(\cdot)$ is differentiable in $\mathbb{R} \backslash\{0\}$, the controlto-state-mapping $u \rightarrow y^{u}$ is not Gâteaux differentiable for $p<2$. This has already been observed in [6]. However, the control-to-state-map is continuous. Indeed, let $\left(u^{k}\right)_{k}$ be a sequence of controls that converges to $u^{0}$ in $\mathbb{R}^{\left|\mathcal{J}_{N}^{S}\right|}$. For the corresponding solutions $y^{k}:=y^{u^{k}}, y^{0}:=y^{u^{0}}$, we obtain

$$
\begin{aligned}
& \left.\left.\sum_{i \in \mathcal{I}} \int_{0}^{\ell_{i}} \mid\left(\mid \beta\left(y_{i}^{k}\right)-\beta\left(y_{i}^{0}\right)\right)\left(y_{i}^{k}-y_{i}^{0}\right)\right)+\beta\left(\partial_{x} y_{i}^{k}\right)-\beta\left(\partial_{x} y_{i}^{0}\right)\right)\left(\partial_{x} y_{i}^{k}-\partial_{x} y_{i}^{0}\right) d x \\
& =\sum_{j \in \mathcal{J}_{N}^{S}} \sum_{i \in \mathcal{I}_{j}}\left(u_{j}^{k}-u_{i}^{0}\right)\left(y_{i}^{k}-y_{i}^{0}\right)\left(n_{j}\right) .
\end{aligned}
$$

By the continuity of $\beta(\cdot)$ and the strong convergence of $y^{k}$ to $y^{0}$ in $V$, we obtain

Theorem 2.2. The mapping $u \rightarrow y^{u}$, where $y^{u}$ solves (1.15) is continuous between $\mathbb{R}^{\left|\mathcal{J}_{N}^{S}\right|}$ and $V$.

\section{Optimal control}

We are now in the position to prove existence of optimal pairs $(y, u)$ for the optimal control problem (1.16). Indeed, the control-to-state-map is continuous and the cost function is strictly convex and lower-semi-continuous with respect to the strong topologies.

Theorem 3.1. The optimal control problem (1.16) admits a unique solution $(\bar{y}, \bar{u}) \in V \times \mathbb{R}^{\left|\mathcal{J}_{N}^{S}\right|}$. 
The proof follows classical arguments and is omitted here. It becomes now important to investigate optimality conditions. Clearly, this involves adjoint states, satisfying the adjoint problem. However, the adjoint problem, in turn, involves the linearization of the state equation along the optimal trajectory. As the nonlinearities of the state equation are governed by the mapping $\beta(\cdot)$ and the derivative of this mapping $\beta^{\prime}(s)=\frac{1}{2 \sqrt{|s|}}$ is unbounded inn the neighbourhood of $s=0$, we need an argument that the optimal solution $y_{i}$ together with its derivative $\partial_{x} y_{i}$ stays away from 0 . We begin with a regularization of the problem, as proposed in $[5,6]$. The regularization is made in order to bring us back into the standard $p=2$ setting.

$$
\begin{aligned}
& \epsilon y_{i}(x)+\alpha_{i} \beta\left(y_{i}\right)(x)-\partial_{x}\left(\epsilon \partial_{x} y_{i}(x)+\beta\left(\partial_{x} y_{i}(x)\right)\right)=f_{i}^{1}(x), i \in \mathcal{I}, x \in\left(0, \ell_{i}\right) \\
& \quad y_{i}\left(n_{j}\right)=y_{k}\left(n_{j}\right), \forall i, k \in \mathcal{I}_{j}, j \in \mathcal{J}^{M} \\
& \quad \sum_{i \in \mathcal{I}_{j}} d_{i j}\left(\epsilon \partial_{x} y_{i}\left(n_{j}\right)+\beta\left(\partial_{x} y_{i}\right)\left(n_{j}\right)=0, j \in \mathcal{J}^{M}\right. \\
& \epsilon \partial_{x} y_{i}\left(n_{j}\right)+\beta\left(\partial_{x} y_{i}\right)\left(n_{j}\right)=u_{j}, d_{j}=1, i \in \mathcal{I}_{j}, j \in \mathcal{J}_{N}^{S} \\
& \quad y_{i}\left(n_{j}\right)=0, i \in \mathcal{I}_{j}, j \in \mathcal{J}_{D}^{S} .
\end{aligned}
$$

We formulate the $\epsilon$ - perturbed optimal control problem as follows.

$$
\begin{aligned}
\min _{\left(y^{\epsilon}, u^{\epsilon}\right)} I_{\epsilon}\left(y^{\epsilon}, u^{\epsilon}\right):= & \sum_{i \in \mathcal{I}} \int_{0}^{\ell_{i}} I_{i}\left(y_{i}^{\epsilon}\right) d x+\frac{\nu}{2} \sum_{j \in \mathcal{J}^{S}}\left|u_{j}^{\epsilon}\right|^{2}+\frac{\nu}{2} \sum_{j \in \mathcal{J}^{S}}\left|u_{j}^{\epsilon}-\bar{u}_{j}\right|^{2} \\
& \text { s.t. } \\
\left(y^{\epsilon}, u^{\epsilon}\right) \text { satisfies } & (3.1),
\end{aligned}
$$

where $\bar{u}$ denotes the optimal control for the unperturbed problem. Problem (3.2) is a standard optimal control problem, where the control-to-state-map is now continuously differentiable. The following theorem, therefore, can be stated without dwelling on the proof.

Theorem 3.2. For each $\epsilon>0$, there exists an optimal pair $y^{\epsilon}, u^{\epsilon}$ and an adjoint state $p^{\epsilon}$ such that $y^{\epsilon}, p^{\epsilon}$ stay in

$$
\begin{aligned}
& W:=\left\{y \in \Pi_{i \in \mathcal{I}} H^{1}\left(0, \ell_{i}\right) \mid y_{i}\left(n_{j}\right)=y_{k}\left(n_{j}\right), i, k \in \mathcal{I}_{j}, j \in \mathcal{J}^{M}, y_{i}\left(n_{j}\right)=0,\right. \\
&\left.i \in \mathcal{I}_{j}, j \in \mathcal{J}_{D}^{S} \cdot\right\}
\end{aligned}
$$


and satisfy the following optimality system.

$$
\begin{aligned}
& \epsilon y_{i}+\alpha_{i} \beta\left(y_{i}\right)-\partial_{x}\left(\epsilon \partial_{x} y_{i}+\beta\left(\partial_{x} y_{i}\right)\right)=f_{i}, i \in \mathcal{I}, x \in\left(0, \ell_{i}\right) \\
& \epsilon p_{i}+\alpha_{i} \beta^{\prime}\left(y_{i}\right) p_{i}-\partial_{x}\left(\epsilon \partial_{x} p_{i}+\beta^{\prime}\left(\partial_{x} y_{i} \partial_{x} p_{i}\right)\right) \\
& \quad=\kappa\left(\left|y_{i}-y_{i}^{d}\right|^{p-2}\left(y_{i}-y_{i}^{d}\right), i \in \mathcal{I}, x \in\left(0, \ell_{i}\right)\right. \\
& \quad y_{i}\left(n_{j}\right)=y_{k}\left(n_{j}\right), \forall i, k \in \mathcal{I}_{j}, p_{i}\left(n_{j}\right)=p_{k}\left(n_{j}\right), \forall i, k \in \mathcal{I}_{j}, j \in \mathcal{J}^{M} \\
& \quad \sum_{i \in \mathcal{I}_{j}} d_{i j}\left(\epsilon \partial_{x} y_{i}\left(n_{j}\right)+\beta\left(\partial_{x} y_{i}\right)\left(n_{j}\right)=0, j \in \mathcal{J}^{M}\right. \\
& \quad \sum_{i \in \mathcal{I}_{j}} d_{i j}\left(\epsilon \partial_{x} p_{i}\left(n_{j}\right)+\beta^{\prime}\left(\partial_{x} y_{i}\right)\left(n_{j}\right) \partial_{x} p_{i}\left(n_{j}\right)=0, j \in \mathcal{J}^{M}\right. \\
& \epsilon \partial_{x} y_{i}\left(n_{j}\right)+\beta\left(\partial_{x} y_{i}\right)\left(n_{j}\right)=u_{j}, d_{j}=1, i \in \mathcal{I}_{j}, j \in \mathcal{J}_{N}^{S} \\
& \epsilon \partial_{x} p_{i}\left(n_{j}\right)+\beta^{\prime}\left(\partial_{x} y_{i}\right)\left(n_{j}\right) \partial_{x} p_{i}\left(n_{j}\right)=0, d_{j}=1, i \in \mathcal{I}_{j}, j \in \mathcal{J}_{N}^{S} \\
& y_{i}\left(n_{j}\right)=0, p_{i}\left(n_{j}\right)=0 i \in \mathcal{I}_{j}, j \in \mathcal{J}_{D}^{S} \\
& p_{i}^{\epsilon}\left(n_{j}\right)+\nu u_{j}^{\epsilon}+\left(u_{j}^{\epsilon}-\bar{u}_{j}\right)=0, i \in \mathcal{I}_{j}, j \in \mathcal{J}_{N}^{S} .
\end{aligned}
$$

In order to understand the limiting procedure as ${ }^{\epsilon} \rightarrow 0$, we look at the adjoint equation and multiply it by $p \epsilon$. We obtain

$$
\begin{aligned}
\int_{0}^{\ell_{i}} \epsilon\left(p_{i}^{\epsilon}\right)^{2} & +\alpha_{i} \beta^{\prime}\left(y_{i}^{\epsilon}\right)\left(p_{i}^{\epsilon}\right)^{2}+\left(\epsilon \partial_{x} p_{i}^{\epsilon}+\beta^{\prime}\left(\partial_{x} y_{i}^{\epsilon}\right) \partial_{x} p_{i}^{\epsilon}\right) \partial_{x} p_{i}^{\epsilon} d x \\
& =\int_{0}^{\ell_{i}}\left|y_{i}^{\epsilon}-y_{i}^{d}\right|^{p-2}\left(y_{i}^{\epsilon}-y_{i}^{d}\right) p_{i}^{\epsilon} d x .
\end{aligned}
$$

If now $y_{i}^{\epsilon} \rightarrow y_{i}$ strongly in $W$, then

$$
\epsilon+\alpha_{i} \beta^{\prime}\left(y_{i}^{\epsilon}\right)=\rightarrow \alpha_{i} \beta^{\prime}\left(y_{i}^{\epsilon}\right), \quad \epsilon+\alpha_{i} \beta^{\prime}\left(\partial_{x} y_{i}^{\epsilon}\right)=\rightarrow \alpha_{i} \beta^{\prime}\left(\partial_{x} y_{i}^{\epsilon}\right)
$$

Moreover, for bounded $\left(y_{i}^{\epsilon}\right)_{\epsilon},\left(p_{i}^{\epsilon}\right)_{\epsilon}$, the terms in the equation stay bounded and if $p_{i}^{\epsilon} \rightarrow p_{i}$ (even weakly), the limiting equation is

$$
\int_{0}^{\ell_{i}} \alpha_{i} \beta^{\prime}\left(y_{i}\right)\left(p_{i}\right)^{2}+\left(\beta^{\prime}\left(\partial_{x} y_{i}\right) \partial_{x} p_{i}\right) \partial_{x} p_{i}^{\epsilon} d x=\int_{0}^{\ell_{i}}\left|y_{i}-y_{i}^{d}\right|^{p-2}\left(y_{i}-y_{i}^{d}\right) p_{i} d x
$$

We are now in the position to show the following limiting result. 
Theorem 3.3. There exists $\bar{p} \in V$ satisfying together with the optimal pair $(\bar{y}, \bar{u})$ the first order optimality condition.

$$
\begin{gathered}
\alpha_{i} \beta\left(\bar{y}_{i}\right)-\partial_{x}\left(\beta\left(\partial_{x} \bar{y}_{i}\right)\right)=f_{i}, i \in \mathcal{I}, x \in\left(0, \ell_{i}\right) \\
\alpha_{i} \beta^{\prime}\left(\bar{y}_{i}\right) \bar{p}_{i}-\partial_{x}\left(\beta^{\prime}\left(\partial_{x} \bar{y}_{i} \partial_{x} \bar{p}_{i}\right)\right)=\kappa\left(\left|\bar{y}_{i}-y_{i}^{d}\right|^{p-2}\left(\bar{y}_{i}-y_{i}^{d}\right), i \in \mathcal{I}, x \in\left(0, \ell_{i}\right)\right. \\
\bar{y}_{i}\left(n_{j}\right)=\bar{y}_{k}\left(n_{j}\right), \forall i, k \in \mathcal{I}_{j}, \bar{p}_{i}\left(n_{j}\right)=\bar{p}_{k}\left(n_{j}\right), \forall i, k \in \mathcal{I}_{j}, j \in \mathcal{J}^{M} \\
\sum_{i \in \mathcal{I}_{j}} d_{i j}\left(+\beta\left(\partial_{x} \bar{y}_{i}\right)\left(n_{j}\right)=0, j \in \mathcal{J}^{M}\right. \\
\sum_{i \in \mathcal{I}_{j}} d_{i j}\left(+\beta^{\prime}\left(\partial_{x} \bar{y}_{i}\right)\left(n_{j}\right) \partial_{x} \bar{p}_{i}\left(n_{j}\right)=0, j \in \mathcal{J}^{M}\right. \\
\beta\left(\partial_{x} \bar{y}_{i}\right)\left(n_{j}\right)=\bar{u}_{j}, d_{j}=1, i \in \mathcal{I}_{j}, j \in \mathcal{J}_{N}^{S} \\
\beta^{\prime}\left(\partial_{x} \bar{y}_{i}\right)\left(n_{j}\right) \partial_{x} \bar{p}_{i}\left(n_{j}\right)=0, d_{j}=1, i \in \mathcal{I}_{j}, j \in \mathcal{J}_{N}^{S} \\
\bar{y}_{i}\left(n_{j}\right)=0, \bar{p}_{i}\left(n_{j}\right)=0 i \in \mathcal{I}_{j}, j \in \mathcal{J}_{D}^{S} \\
\bar{p}_{i}^{\epsilon}\left(n_{j}\right)+\nu \bar{u}_{j}=0, i \in \mathcal{I}_{j}, j \in \mathcal{J}_{N}^{S}
\end{gathered}
$$

Example. We consider the tripod above, where we use $\alpha_{i}=\alpha=10, f_{i}=$ $f=10$ and demonstrate the effect of the second order nonlinear operator by choosing coefficients $\gamma_{i}=\gamma=1$ or 100 . The penalty for $\kappa_{i}=\kappa$ is chosen equal to 1000 . For the calculations, we used a regularization, as in the text as $\epsilon=0.01$. The numerical studies reveal that the system behaviour does not largely depend on $\epsilon$ as long this number is in order of magnitude smaller than the resolution tolerance which was set here to $1 e-4$. The optimality system is solved using the MATLAB routine bvp $4 c$. See Fig. 3.1 and Fig. 3.2 .

\section{References}

[1] R. Alonso, M. Santillana, and C. Dawson. On the diffusive wave approximation of the shallow water equations. European J. Appl. Math., 19(5):575-606, 2008.

[2] A. Bamberger, M. Sorine, and J. P. Yvon. Analyse et contrôle d'un réseau de transport de gaz. In Computing methods in applied sciences and engineering (Proc. Third Internat. Sympos., Versailles, 1977), II, volume 91 of Lecture Notes in Phys., pages 347-359. Springer, Berlin-New York, 1979.

[3] Alain Bamberger. étude d'une équation doublement non linéaire. J. Functional Analysis, 24(2):148-155, 1977.

[4] J. Brouwer, I. Gasser, and M. Herty. Gas pipeline models revisited: Model hierarchies, nonisothermal models, and simulations of networks. 9(2):601623. 

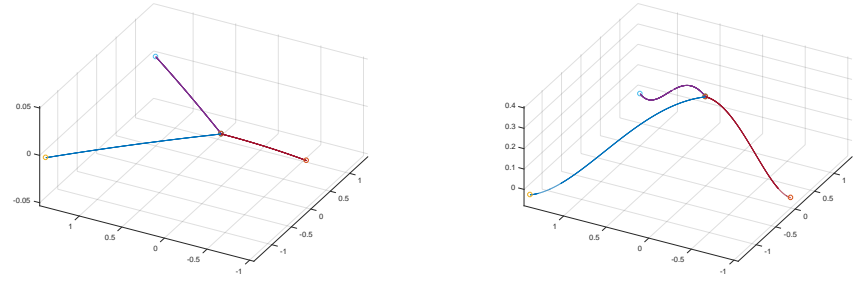

Figure 3. left: $\alpha=10, \gamma=1, f_{i}=10$; right: $\alpha=10$, $\gamma=100, f_{i}=10$
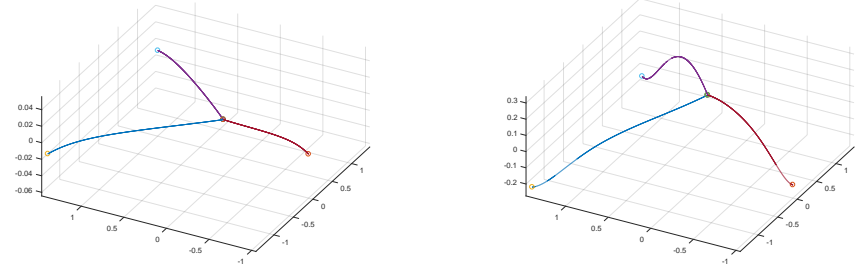

Figure 4. left: $\alpha=100, \gamma=1, f_{i}=10$; right: $\alpha=1$, $\gamma=0.1, f_{i}=10$ 
[5] Eduardo Casas and Luis A. Fernández. Optimal control of quasilinear elliptic equations. In Control of partial differential equations (Santiago de Compostela, 1987), volume 114 of Lect. Notes Control Inf. Sci., pages 92-99. Springer, Berlin, 1989.

[6] Eduardo Casas and Luis Alberto Fernández. Optimal control of quasilinear elliptic equations with nondifferentiable coefficients at the origin. Rev. Mat. Univ. Complut. Madrid, 4(2-3):227-250, 1991.

[7] P. I. Kogut and G. Leugering. Optimal Control Problems for Partial Differential Equations on Reticulated Domains. Systems and Control: Foundations and Applications. Springer.

[8] G. Leugering and G. Mophou. Optimal control of a doubly nonlinear parabolic equation on a metric graph. in preparation 2017.

[9] Randall J. Le Veque. Finite Volume Methods for Hyperbolic Problems. Cambridge University Press.

[10] Randall J. Le Veque. Numerical Methods for Conservation Laws. BirkhÃd'user.

[11] Tomá s Roubí ček. Nonlinear partial differential equations with applications, volume 153 of International Series of Numerical Mathematics. Birkhäuser/Springer Basel AG, Basel, second edition, 2013.

[12] Joel Smoller. Shock Waves and Reaction-Diffusion Equations, volume 258 of Grundlehren der mathematischen Wissenschaften. Springer Verlag.

Günter Leugering

Lehrstuhl Angewandte Mathematik Lehrstuhl II Universität Erlangen-Nürnberg Cauerstr. 11 D-91058 Erlangen, Germany

e-mail: guenter.leugering@fau.de

Gisèle Mophou

Humboldt Research Chair A.I.M.S. Cameroon, Limbé

e-mail: gisele.mophou@aims-cameroon.org 